\title{
Alimentação do recém-nascido pré-termo: aleitamento materno, copo e mamadeira
}

\author{
Feeding preterm infants: breast, cup and bottle
}

\author{
Andréa Monteiro Correia Medeiros ${ }^{1}$, Aretha Tatiane Bernardi ${ }^{2}$
}

\begin{abstract}
RESUMO
Objetivo: Verificar a oferta de seio materno em bebês nascidos pré-termos, internados na Unidade de Terapia Intensiva do Hospital e Maternidade Neomater, relacionando este dado com a forma de oferecer a dieta (copo ou mamadeira), na ausência da mãe, e a estimulação fonoaudiológica realizada. Métodos: Participaram 48 recém-nascidos prematuros, com idade gestacional inferior ou igual a 36 6/7 semanas, com peso $\leq 2500$ gramas e padrão respiratório estável. Os recém-nascidos foram divididos em dois grupos: grupo A (35 recém-nascidos) com dieta por mamadeira e grupo B (13 recém-nascidos) com dieta por copo. O acompanhamento fonoaudiológico foi organizado em Fase 1 (sucção não-nutritiva em "dedo enluvado" ou "mama vazia" concomitante a dieta por sonda); Fase 2 (oferta de dieta via oral - seio materno, copo ou mamadeira - com necessidade de complemento por sonda); Fase 3 (oferta de dieta via oral exclusiva, em seio materno, copo ou mamadeira); Fase 4 (oferta de seio materno efetivo). Foi realizado comparativo entre os grupos quanto ao número de dias que permaneceu em cada fase. Os dados foram tratados estatisticamente com o teste t-independente, com nível de significância de 5\%. Resultados: Não houve diferenças significativas entre os grupos para nenhum dos parâmetros estudados. Houve igual aceitação do seio materno pelos recém-nascidos, tanto no grupo copo como no grupo mamadeira. Conclusão: O aleitamento materno pode ser igualmente aceito, independentemente da forma de oferta da dieta (copo ou mamadeira), desde que haja o adequado acompanhamento fonoaudiológico e o incentivo ao aleitamento materno em idade precoce.
\end{abstract}

Descritores: Aleitamento materno; Mamadeira; Terapia intensiva neonatal; Dieta; Alimentação artificial; Métodos de alimentação; Recém-nascido; Prematuro

\section{INTRODUÇÃO}

O aleitamento materno é fundamental para o adequado desenvolvimento motor-oral e o estabelecimento correto das funções estomatognáticas. Fazem parte deste sistema as estruturas orais estáticas e dinâmicas que, equilibradas e controladas pelo sistema nervoso central, serão responsáveis pelo funcionamento harmônico da face ${ }^{(1)}$.

Ao sugar o seio materno, o recém-nascido faz esforço com os músculos da face para extrair o leite do peito, o que estimula, desenvolve e fortalece suas estruturas orais (lábios, língua, bochechas, ossos e músculos da face $)^{(2)}$.

$\mathrm{O}$ recém-nascido $(\mathrm{RN})$ tende a responder diferentemen-

Trabalho realizado no Hospital e Maternidade Neomater - São Bernardo do Campo (SP), Brasil.

(1) Núcleo de Fonoaudiologia, Universidade Federal de Sergipe - UFS - São Cristóvão (SE), Brasil.

(2) Programa de Especialização em Motricidade Orofacial, Hospital e Maternidade Neomater - São Bernardo do Campo (SP), Brasil.

Endereço para correspondência: Andréa Monteiro Correia Medeiros. Universidade Federal de Sergipe - UFS. Centro de Ciências Biológicas e da Saúde - CCBS - Núcleo de Fonoaudiologia. R. Marechal Rondon, s/n, Cidade Universitária Prof. José Aloísio de Campos, Jd. Rosa Elze, São Cristóvão (SE), Brasil, CEP: 49100-000. E-mail: andreamcmedeiros@ig.com.br

Recebido em: 22/3/2010; Aceito em: 21/7/2010 te às diversas estimulações, de acordo com determinadas motivações. Assim, responderá de um modo positivo a um estímulo que lhe seja apropriado, e poderá realizar movimentos de repulsa ou apresentar comportamentos de proteção diante de estímulos considerados nocivos ou invasivos ao seu organismo ${ }^{(3)}$.

Atualmente, em alguns casos especiais, quando o bebê não pode ser alimentado no seio materno, temporária ou permanentemente, é utilizado, em alguns hospitais, o bico artificial para oferta da dieta, buscando que o bebê melhore o padrão de sucção. $\mathrm{O}$ uso da mamadeira pretende treinar a coordenação das funções de sucção, deglutição e respiração, quando o RN ainda não tem condições adequadas de Sistema Sensório Motor Oral (SSMO) para a prática do aleitamento natural ${ }^{(4)}$.

Embora a sucção seja um comportamento reflexo, ela pode ser intensificada ou modificada de acordo com as experiências obtidas. Os comportamentos miofuncionais orais esperados na sucção do neonato são: lábios vedados, compressão labial e formação de leve sulco nas comissuras labiais, movimentação dos músculos masseteres e movimentos mandibulares e ântero-posteriores da língua ${ }^{(5)}$. Alterações antes, durante e/ou após o nascimento, podem comprometer a alimentação e o desenvolvimento neuropsicomotor do bebê $\hat{e}^{(6)}$. 
A mamadeira, depois do aleitamento materno, é a segunda forma mais conhecida pela população em geral para oferecer o alimento ao RN. Os bicos artificiais, dentre tantas formas de oferta de dietas, foram criados como uma opção de aleitamento e de auxílio aos bebês e aos pais.

Para alguns profissionais, as dificuldades no aleitamento materno, secundárias ao uso de mamadeiras e/ou chupetas, podem gerar a "confusão de bicos", devido às diferenças existentes entre a sucção na mama e no bico $\operatorname{artificial}^{(7)}$.

Por outro lado, alguns autores questionam a existência de confusão de bicos, pois não existem evidências que comprovem que o recém-nascido apresenta uma habilidade limitada para se adaptar a várias configurações orais ${ }^{(8)}$.

Assim, enquanto alguns fonoaudiólogos indicam o uso de bico ortodôntico na adequação da musculatura orofacial nos casos em que não é viável o aleitamento materno em seio materno exclusivo, outros profissionais consideram que o uso de bicos artificiais pode ser prejudicial ao bebê.

Por outro lado, mesmo os fonoaudiólogos que optam pela indicação da mamadeira com bico ortodôntico têm alertado para o fato de que o uso prolongado desta, ou seja, a partir da dentadura decídua instalada, pode acarretar grande incidência de mordida aberta anterior.

Na prática clínica, observa-se que frequentemente pacientes com mordida aberta anterior são encaminhados, antes da correção ortodôntica, para a fonoterapia, o que aponta para a necessidade da retirada da mamadeira em idade precoce ${ }^{(1)}$.

Segundo recomendação da Organização Mundial de Saúde (OMS), foi criada a Iniciativa Hospital Amigo da Criança (IHAC), que estabelece "Dez passos para o sucesso do aleitamento materno", dentre esses dez passos, o nono diz: "Não dar bicos artificiais ou chupetas para crianças amamentadas ao seio"(9). O motivo da "proibição" do aleitamento por mamadeira é a suposta "confusão de bicos" adquirida pelo bebê, ou seja, o lactente apresentará dificuldades para sugar no seio materno (SM), pois aprendeu a sugar no bico artificial.

A alimentação no copo tem sido utilizada há anos em países em desenvolvimento, como objetivo de proporcionar um método de alimentação seguro para o recém-nascido, especialmente quando os meios de esterilização de mamadeiras não são acessíveis ${ }^{(6,9-11)}$.

Entretanto, o copo é visto por vários fonoaudiólogos como um utensílio que acarreta muitos problemas na administração da dieta para o recém-nascido, tais como: escape e desperdício de leite, falta do vedamento labial anterior, aumento de risco de broncoaspiração, diminuição da estimulação da musculatura da sucção (músculos bucinadores), entre outros.

A fisiologia de aceitação da dieta por copo é bem diferente da sucção realizada pelos recém-nascidos, seja no seio materno ou na mamadeira, ocorrendo o risco de deglutição atípica no futuro. Considera-se deglutição atípica quando existe alteração nos mecanismos da contração da musculatura envolvida nesse processo ${ }^{(12)}$.

Desta forma, existem hoje diferentes técnicas para a alimentação do recém-nascido prematuro, em que há, entre os profissionais de saúde, o constante debate sobre se a utilização do bico artificial ou do copo prejudica a aceitação do seio materno.
Qualquer técnica alternativa de oferta de dieta para o recém-nascido deve objetivar o treino de habilidades para a aceitação da amamentação natural, a qual estimula a neuromusculatura facial da criança, o que induz ao crescimento harmônico de todas as estruturas craniofaciais ${ }^{(13)}$.

Preconiza-se inclusive que os serviços de saúde pratiquem o método mãe canguru que, ao possibilitar o contato precoce pela pele da mãe com o bebê prematuro, contribui significativamente para a melhora na produção de leite pela mãe, favorecendo o aleitamento materno futuro, mesmo quando esta criança precisar ser alimentada por sonda, copo ou mamadeira em algum momento da internação ${ }^{(14-16)}$.

Neste sentido, este trabalho teve como objetivo verificar se, no momento da alta hospitalar, o RN apresenta plenas condições de receber a dieta no SM, e relacionar este dado à estimulação realizada e ao tipo de oferta de dieta oferecido (copo ou mamadeira) durante sua internação.

\section{MÉTODOS}

Esta pesquisa foi realizada na Unidade de Cuidado Intensivo Neonatal (UCIN) do Hospital e Maternidade Neomater, situada na cidade de São Bernardo do Campo, a partir de autorizações da Diretoria Clínica e Comissão de Ética do respectivo hospital e do Comitê de Ética em Pesquisa da Faculdade de Medicina do ABC (processo de no. 318/2009).

\section{Sujeitos}

Fizeram parte da amostra desta pesquisa 48 recémnascidos pré-temo (RNPT), com idade gestacional inferior ou igual a 36 6/7 semanas (de acordo com a Organização Mundial de Saúde - OMS), que foram recrutados a partir coleta de dados no prontuário médico.

Como critérios de inclusão para ambos os grupos foram selecionados RNPT com peso inferior ou igual a 2500 gramas, com idade gestacional inferior ou igual a 36 6/7 semanas, estáveis clinicamente e recebendo dieta (leite materno ou fórmula) prescrita pela equipe médica. Como critérios de exclusão, não participaram recém-nascidos portadores de síndromes genéticas e mal-formativas, afecções ósteomio-articulares não resolvidas (infecções, fraturas e lesões nervosas periféricas) ou qualquer anormalidade neurológica evidenciada através de exame de ultra-som craniano (hemorragia peri-intraventricular - HPIV graus III e IV, hidrocefalia, encefalopatia hipóxico-isquêmica - EHI, leucomalácia peri ventricular e outras).

Os bebês foram divididos em dois grupos:

- Grupo A: administração da dieta por mamadeira (35 recémnascidos);

- Grupo B: administração de dieta por copo (13 recémnascidos).

Até o momento da alimentação por via oral (VO), o recém-nascido já poderia ter sido estimulado com a técnica de sucção não-nutritiva ( $\mathrm{SNN}$ ) - estimulação com "dedo enluvado" concomitante à oferta da dieta via sonda e/ou técnica de relactação - estimulação com "mama vazia" concomitante à oferta de dieta via sonda. 


\section{Procedimentos}

Inicialmente, foi realizado o levantamento dos prontuários dos recém-nascidos internados na Unidade de Cuidado Intensivo Neonatal (UCIN).

A verificação desses prontuários foi feita por fonoaudiólogas pertencentes ao grupo responsável pela pesquisa, sendo registrados os dados dos RN's quanto ao gênero, idade gestacional corrigida, e número de dias que permaneceu em cada fase da intervenção fonoaudiológica (Fase 1: sucção nãonutritiva; Fase 2: dieta por sonda mais complemento por via oral; Fase 3: via oral exclusiva; Fase 4: aleitamento materno exclusivo e efetivo) (Quadro 1).

A intervenção fonoaudiológica foi registrada no Protocolo

Quadro 1. Caracterização dos RN's quanto ao sexo, idade gestacional, idade gestacional corrigida, e número de dias que permaneceu em cada fase da intervenção fonoaudiológica

\begin{tabular}{|c|c|c|c|c|c|c|c|}
\hline \multirow[b]{2}{*}{$\mathrm{N}^{\circ} \mathrm{RN}$} & \multirow[b]{2}{*}{ Sexo } & \multirow[b]{2}{*}{ IG } & \multicolumn{3}{|c|}{$\mathrm{N}^{\circ}$ de dias na } & & \multirow{2}{*}{$\begin{array}{c}\mathrm{N}^{\circ} \text { de dias na } \\
\text { Fase } 4\end{array}$} \\
\hline & & & IGC & Fase 1 & $\mathrm{~N}^{\circ}$ de dias na Fase 2 & $\mathrm{~N}^{\circ}$ de dias na Fase 3 & \\
\hline 1 & $M$ & 28 & 37 & 1 & $3(\mathrm{SM})$ & 1 (Сopo) & 1 \\
\hline 2 & M & 32 & $346 / 7$ & 2 & 4 (VOBC) & 1 (VOBO) & 1 \\
\hline 3 & $\mathrm{~F}$ & $341 / 7$ & $344 / 7$ & 1 & 12 (VOBO) & 2 (VOBOPT) & 0 \\
\hline 4 & M & 33 & $343 / 7$ & 5 & 3 (VOBC) & 5 (VOBC) & 1 \\
\hline 5 & M & 30 & $304 / 7$ & 8 & 0 & 1 (VOBC) 2 (VOBO) & 3 \\
\hline 6 & M & 32 & $336 / 7$ & 3 & 6 (Соро) & 4 (Соpo) & 0 \\
\hline 7 & M & 33 & $342 / 7$ & 3 & 0 & 1 (Соро) & 3 \\
\hline 8 & $\mathrm{~F}$ & 28 & $381 / 7$ & 5 & 6 (VOBC) & $1(\mathrm{VOBC})$ & 1 \\
\hline 9 & M & 33 & $346 / 7$ & 2 & 0 & 1 (VOBC) 1 (VOBO) & 2 \\
\hline 10 & M & 28 & $336 / 7$ & 2 & 8 (VOBC) & 1 (VOBC) & 1 \\
\hline 11 & M & 34 & 35 & 2 & 0 & 3 (Copo) & 2 \\
\hline 12 & $\mathrm{~F}$ & 33 & $351 / 7$ & 2 & 4 (VOBC) & 5 (VOBC) & 10 \\
\hline 13 & $\mathrm{~F}$ & 33 & $346 / 7$ & 1 & 1 (VOBC) & 4 (VOBC) & 0 \\
\hline 14 & $\mathrm{~F}$ & 31 & $341 / 7$ & 2 & 3 (VOBC) & 1 (VOBC) 2 (VOBO) & 1 \\
\hline 15 & $\mathrm{~F}$ & 35 & $362 / 7$ & 1 & $1(\mathrm{SM})$ & 0 & 2 \\
\hline 16 & $\mathrm{~F}$ & 32 & 33 & 2 & $1(\mathrm{SM})$ & 2 (VOBC) & 2 \\
\hline 17 & $M$ & 34 & 35 & 4 & 0 & 4 (Соро) & 3 \\
\hline 18 & $\mathrm{~F}$ & 35 & $352 / 7$ & 1 & 0 & 2 (Соро) & 3 \\
\hline 19 & M & 35 & $361 / 7$ & 2 & 0 & 6 (VOBC) & 6 \\
\hline 20 & $M$ & 32 & $335 / 7$ & 3 & 1 (SM) & 1 (VOBC) & 1 \\
\hline 21 & $M$ & 31 & $315 / 7$ & 10 & 0 & 6 (VOBC) & 3 \\
\hline 22 & $\mathrm{~F}$ & $364 / 7$ & $371 / 7$ & 4 & 2 (VOBC) & 3 (VOBC) & 7 \\
\hline 23 & M & 28 & $403 / 7$ & 1 & 0 & 5 (VOBC) 1 (VOBO) & 1 \\
\hline 24 & $\mathrm{~F}$ & 27 & 35 & 5 & 2 (VOBC) & 2 (VOBC) 1 (VOBO) & 1 \\
\hline 25 & M & 28 & 41 & 3 & 2 (VOBC) & 1 (VOBC) 3 (VOBO) & 1 \\
\hline 26 & $\mathrm{~F}$ & $284 / 7$ & 31 & 6 & 7 (VOBC) & 4 (VOBC) 1 (VOBO) & 1 \\
\hline 27 & $M$ & 35 & $364 / 7$ & 1 & 1 (Copo) 1 (SM) & 1 (Соpo) & 1 \\
\hline 28 & $\mathrm{~F}$ & 30 & $303 / 7$ & 8 & 8 (VOBC) & 1 (VOBC) 3 (VOBO) & 1 \\
\hline 29 & $\mathrm{~F}$ & 30 & $451 / 7$ & 5 & 2 (Соро) & 3 (Соро) & 2 \\
\hline 30 & $M$ & 30 & $344 / 7$ & 1 & 1 (VOBC) & 1 (VOBC) 1 (VOBO) & 1 \\
\hline 31 & $M$ & 32 & $342 / 7$ & 8 & 2 (VOBC) & 1 (VOBC) & 1 \\
\hline 32 & $\mathrm{~F}$ & 29 & $351 / 7$ & 3 & 0 & 3 (Copo) & 7 \\
\hline 33 & $\mathrm{~F}$ & 29 & $363 / 7$ & 9 & $2(\mathrm{SM})$ & 2 (Соро) & 3 \\
\hline 34 & $\mathrm{~F}$ & 33 & 34 & 2 & $3(\mathrm{SM})$ & 1 (Соро) & 3 \\
\hline 35 & M & 30 & 38 & 1 & 1 (Copo) 5 (SM) & 0 & 1 \\
\hline 36 & M & 33 & $335 / 7$ & 3 & 4 (VOBC) & 3 (VOBC) 2 (VOBO) & 1 \\
\hline 37 & $\mathrm{~F}$ & 32 & $336 / 7$ & 1 & 6 (VOBC) & 1 (VOBO) & 1 \\
\hline 38 & M & 32 & $325 / 7$ & 1 & 3 (VOBC) & 2 (VOBOPT) 2 (VOBO) & 2 \\
\hline 39 & $\mathrm{~F}$ & 31 & 34 & 1 & 1 (VOBC) & $1(\mathrm{VOBC}) 1$ (VOBO) & 1 \\
\hline 40 & M & 34 & $352 / 7$ & 1 & 4 (VOBC) & 3 (VOBC) 1 (VOBO) & 1 \\
\hline 41 & $\mathrm{~F}$ & 32 & 34 & 1 & 1(VOBC) 2(VOBO) & 6 (VOBO) & 1 \\
\hline 42 & $\mathrm{~F}$ & 31 & 33 & 2 & 1 (VOBC) 5(VOBO) & 1 (VOBC) 1 (VOBO) & 1 \\
\hline 43 & M & 31 & 34 & 1 & 5 (VOBC) & 2 (VOBC) 2 (VOBO) & 1 \\
\hline 44 & M & 32 & $336 / 7$ & 1 & 9 (VOBC) & 1 (VOBC) & 1 \\
\hline 45 & M & $335 / 7$ & $345 / 7$ & 6 & 5 (Copo) 2 (SM) & 1 (Copo) & 1 \\
\hline 46 & $\mathrm{~F}$ & 36 & $372 / 7$ & 1 & 3 (VOBC) & 3 (VOBOPT) & 2 \\
\hline 47 & $\mathrm{~F}$ & 35 & $381 / 7$ & 1 & 0 & 3 (VOBOPT) & 1 \\
\hline 48 & M & 30 & 31 & 10 & 2 (VOBC) & 1 (VOBC) & 1 \\
\hline
\end{tabular}

Legenda: $\mathrm{N}$ = número; $\mathrm{RN}$ = recém-nascido; $\mathrm{F}$ = Feminino; $\mathrm{M}=$ Masculino; $\mathrm{IG}$ = idade gestacional; $\mathrm{IGC}$ = idade gestacional corrigida no dia da avaliação fonoaudiológica; SM = Seio Materno; VOBC = Via oral mamadeira com bico comum; VOBO = Via oral mamadeira com bico ortodôntico; VOBOPT = Via oral mamadeira com bico ortodôntico para prematuro 
Tabela 1. Número de dias que os sujeitos permaneceram em cada fase de intervenção fonoaudiológica

\begin{tabular}{|c|c|c|c|c|c|c|}
\hline & & \multicolumn{3}{|l|}{ Grupo } & \multirow{2}{*}{ Valor de $p$} & \multirow{2}{*}{ Resultado } \\
\hline & & Copo & Mamadeira & Total & & \\
\hline & Média & 3,15 & 3,09 & 3,10 & & \\
\hline \multirow[t]{3}{*}{ Fase 1} & DP & 2,38 & 2,76 & 2,64 & 0,938 & copo=mamadeira \\
\hline & $\mathrm{N}$ & 13 & 35 & 48 & & \\
\hline & Média & 2,38 & 3,26 & 3,02 & & \\
\hline \multirow[t]{3}{*}{ Fase 2} & DP & 2,53 & 2,96 & 2,85 & 0,352 & copo $=$ mamadeira \\
\hline & $\mathrm{N}$ & 13 & 35 & 48 & & \\
\hline & Média & 2,00 & 3,03 & 2,75 & & \\
\hline \multirow[t]{3}{*}{ Fase 3} & DP & 1,29 & 1,76 & 1,69 & 0,061 & copo=mamadeira \\
\hline & $\mathrm{N}$ & 13 & 35 & 48 & & \\
\hline & Média & 2,31 & 1,77 & 1,92 & & \\
\hline \multirow[t]{3}{*}{ Fase 4} & $\mathrm{DP}$ & 1,75 & 2,00 & 1,93 & 0,399 & copo=mamadeira \\
\hline & $\mathrm{N}$ & 13 & 35 & 48 & & \\
\hline & Média & 9,85 & 11,14 & 10,79 & & \\
\hline \multirow[t]{2}{*}{ Total } & DP & 3,69 & 4,53 & 4,32 & 0,361 & copo=mamadeira \\
\hline & $\mathrm{N}$ & 13 & 35 & 48 & & \\
\hline
\end{tabular}

Teste $t$ independente $(p<0,05)$

Legenda: $\mathrm{N}$ = número de sujeitos; $\mathrm{DP}$ = desvio-padrão

de Avaliação e Acompanhamento Fonoaudiológico do Hospital e Maternidade Neomater (Anexo 1).

Os RNPT foram divididos em oferta de dieta no copo e na mamadeira, aleatoriamente.

Para a oferta da dieta por mamadeira o RNPT foi colocado em posição elevada, com apoio (no colo ou dentro da incubadora). Os RNPT geralmente receberam a oferta inicial de mamadeira com bico convencional/bico comum, sendo que quando houve indicação de bico ortodôntico, usaram o bico ortodôntico pré-termo (marca NUK®). A dieta por mamadeira foi prescrita e orientada pela fonoaudióloga responsável e nos horários em que o serviço de Fonoaudiologia não esteve presente, a oferta foi ministrada pela enfermeira ou auxiliar de enfermagem previamente orientada e treinada.

Para a oferta de dieta por copo o RNPT também foi colocado em posição elevada, com apoio (no colo ou dentro da incubadora). Inicialmente, pesou-se uma compressa que foi colocada como babador no RN. O bebê, ao sentir o líquido, sorveu ou lambeu o leite, deglutindo até ficar satisfeito. Ao final da oferta, pesou-se novamente a compressa, com o intuito de verificar a quantidade de escape de leite ocorrido. A dieta por copo foi prescrita pela fonoaudióloga responsável e, nos horários em que o serviço de Fonoaudiologia não esteve presente, a oferta foi ministrada pela enfermeira ou auxiliar de enfermagem previamente orientada e treinada.

Durante ambas as ofertas, foram observados e registrados todos os aspectos importantes para a pesquisa, seguindo os itens do protocolo de avaliação e acompanhamento fonoaudiológico.

\section{RESULTADOS}

Os resultados obtidos neste estudo evidenciaram a eficácia da mamada em seio materno (SM), correlacionada à estimula- ção fonoaudiológica realizada e às diferentes formas de oferta de dieta por VO (copo ou mamadeira).

Os resultados foram trabalhados estatisticamente utilizando o teste t-independente ${ }^{(17)}$, com nível de significância de $5 \%$, a fim de comparar os dados quantitativos de dois grupos contendo informações com níveis de mensuração numérica e amostras independentes. Com o intuito de facilitar a visualização dos resultados, as tabelas e as figuras apresentam a média, o desvio padrão e o $\mathrm{N}$ em cada item avaliado durante o estudo.

Quanto ao comparativo entre os grupos, em relação ao número de dias em que cada um permaneceu em cada fase de intervenção fonoaudiológica, os resultados demonstraram que não houve diferenças entre os grupos para nenhuma das fases (Tabela 1).

Embora não tenham existido diferenças entre os grupos para nenhuma das fases do estudo, houve uma tendência à diferença na fase 3 , no sentido de menor número de dias, para copo (Figura 1, Tabela 2).

Com relação ao tempo total da intervenção, foi observada

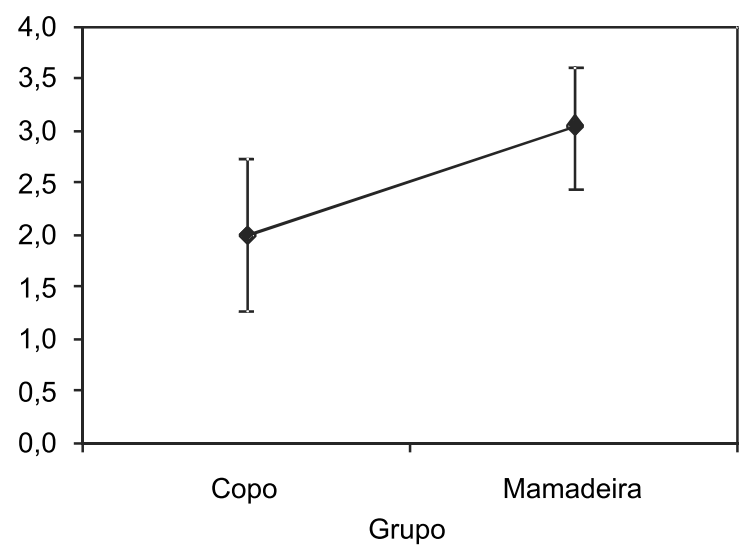

Figura 1. Número de dias de intervenções fonoaudiológicas na Fase 3 
Tabela 2. Número de dias que cada grupo permaneceu na Fase 3

\begin{tabular}{lcc}
\hline & \multicolumn{2}{c}{ Grupo } \\
& Copo & Mamadeira \\
\hline Média & 2,00 & 3,03 \\
DP & 1,29 & 1,76 \\
$\mathrm{~N}$ & 13 & 35 \\
Valor de $\mathrm{p}$ & 0,7 & 0,6 \\
\hline
\end{tabular}

Teste $t$ independente $(p<0,05)$

Legenda: $\mathrm{N}$ = número de sujeitos; $\mathrm{DP}$ = desvio-padrão

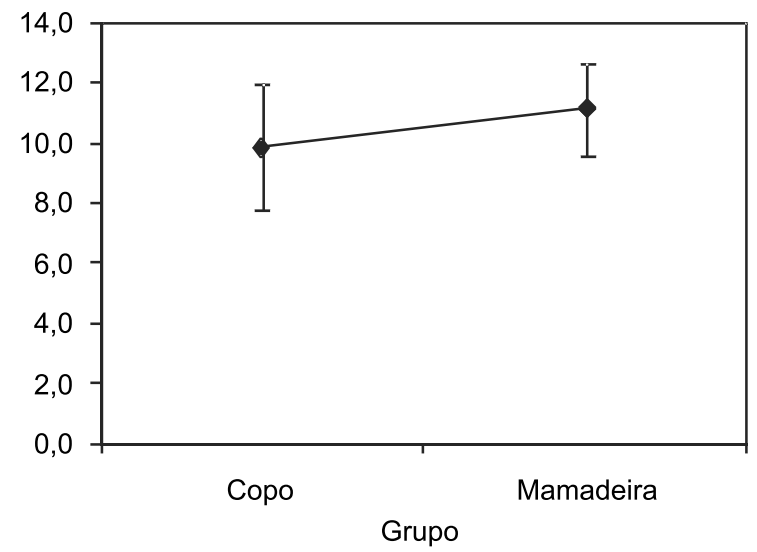

Figura 2. Tempo total de dias de intervenções fonoaudiológicas (somatória de todas as fases)

Tabela 3. Número total de dias de intervenções fonoaudiológicas (somatória de todas as fases)

\begin{tabular}{lcc}
\hline & \multicolumn{2}{c}{ Grupo } \\
& Copo & Mamadeira \\
\hline Média & 9,85 & 11,14 \\
DP & 3,69 & 4,53 \\
$\mathrm{~N}$ & 13 & 35 \\
Valor de $\mathrm{p}$ & 2,1 & 1,5 \\
\hline
\end{tabular}

Teste $t$ independente $(p<0,05)$

Legenda: $\mathrm{N}$ = número de sujeitos; $\mathrm{DP}$ = desvio-padrão

a média de 11 dias, sendo as médias de três dias nas fases 1 , 2 e 3 e de dois dias na fase 4 (Figura 2, Tabela 3).

\section{DISCUSSÃO}

Considerando os achados relacionados à caracterização dos grupos copo e mamadeira, os resultados evidenciam que não houve diferença significativa em nenhum dos parâmetros estudados.

No que tange aos resultados da comparação do número de dias em que os grupos copo e mamadeira permaneceram em cada fase da intervenção fonoaudiológica, não houve di- ferenças entre os grupos para nenhuma das fases. No entanto, observou-se que há uma tendência à diferença na fase 3 , no sentido de menos dias para copo. Tal tendência pode estar relacionada ao fato de que no grupo copo a idade gestacional corrigida (IGC) no dia da avaliação fonoaudiológica era maior do que a do grupo mamadeira, o que pode ter contribuído para a aceitação mais rápida da dieta por via oral em tal grupo.

Neste estudo, o tempo total da intervenção fonoaudiológica com os RNPT com dificuldade na alimentação foi em média de 11 dias, sendo de três dias para cada uma das fases 1,2 e 3 e de dois dias para a fase 4 .

Além do tempo de intervenção fonoaudiológica ter sido semelhante em ambos os grupos, os resultados demonstram que os RNPT, tanto do grupo copo quanto do grupo mamadeira, com acompanhamento fonoaudiológico adequado, conseguiram alimentar-se efetivamente por via oral, sobretudo com aceitação do seio materno, sugerindo que a sucção pode ser intensificada ou modificada, de acordo com as experiências obtidas $^{(6)}$.

Esse dado aponta para o questionamento do conceito que preconiza a não oferta de bicos artificiais para crianças amamentadas ao seio, devido à suposta "confusão de bicos", que fará com que o lactente apresente dificuldades para sugar o seio materno pelo fato que aprendeu a sugar o bico artificial.

Por outro lado, se a utilização do bico artificial não impediu a aceitação do seio materno, é importante salientar que o bico ortodôntico poderia contribuir na estimulação dos músculos orofaciais envolvidos na sucção, sobretudo quanto ao mecanismo dos músculos bucinadores.

Assim, embora haja controvérsias sobre as diferentes técnicas de alimentação do recém-nascido, sugerindo ora a oferta do copo $^{(6,9-11)}$, ora a oferta da mamadeira ${ }^{(4,6)}$, o que parece fundamental é que ambas as técnicas foram efetivas para a facilitação e indicação do aleitamento materno nos RNPT submetidos a um programa de acompanhamento fonoaudiológico direcionado às questões alimentares.

Assim, é fundamental que as famílias sejam amplamente esclarecidas sobre as implicações dos diferentes tipos de dieta na saúde de seu bebê, compreendendo que a realização de um programa de orientação significa a oportunidade de receber informação especializada ${ }^{(18)}$.

\section{CONCLUSÃO}

Considerando ser o aleitamento materno a forma mais saudável e segura de alimentar um bebê, esta pesquisa contribuiu para elucidar que as diferentes técnicas e perspectivas teóricas sobre a forma de oferta de dieta (copo ou mamadeira), quando orientadas de modo ético e criterioso, podem contribuir e auxiliar na realização do aleitamento materno efetivo e na saúde geral do bebê. 


\begin{abstract}
Purpose: To assess the offer of the maternal breast in preterm babies hospitalized at the Intensive Care Unit of the Hospital and Maternity Neomater, linking this datum with the manner to feed them (cup or bottle) in the absence of the mother, and the speechlanguage stimulation carried out. Methods: Forty-eight newborn preterm infants with gestational age less than or equal to 36 6/7 weeks, weighting $\leq 2500$ grams and with stable respiratory pattern participated in this study. The newborns were divided into two groups: group A (35 newborns) with bottle feeding, and group B (13 newborns) with cup feeding. Speech-Language Pathology monitoring was organized in Stage 1 (non-nutritive sucking in "gloved finger" or "empty breast" concomitant with enteral feeding); Stage 2 (oral feeding offer - maternal breast, bottle or cup - with complement provided by enteral feeding); Stage 3 (exclusive oral feeding offer - maternal breast, bottle or cup); Stage 4 (effective breastfeeding offer). A comparison was carried out between the groups regarding the number of days in each phase. Data were statistically analyzed using the independent t-test, with significance level of 5\%. Results: There were no significant differences between the groups for any of the studied parameters. Breast feeding was equally accepted by newborns in the cup and the bottle groups. Conclusion: Breast feeding can be equally accepted, regardless the alternative feeding form used (cup or bottle), provided that there is adequate Speech-Language Pathology monitoring and encouragement to breastfeeding at an early age.
\end{abstract}

Keywords: Breast feeding; Bottle feeding; Intensive care, neonatal; Diet; Artificial feeding; Feeding methods; Infant, newborn; Premature

\section{REFERÊNCIAS}

1. Varandas CPM, Campos LG, Motta AR. Adesão ao tratamento fonoaudiológico segundo a visão de ortodontistas e odontopediatras. Rev Soc Bras Fonoaudiol. 2008;13(3):233-9.

2. Pivante $\mathrm{CM}$, Medeiros AMC. Intervenções fonoaudiológicas no aleitamento materno junto às mães de paridade zero. Mundo Saúde (1995). 2006;30(1):87-95.

3. Medeiros AMC. A existência de sistema sensório-motor integrado em recém-nascidos humanos. Psicol USP. 2007;18(2):11-33.

4. Medeiros AMC, Nery VGC, Simplício M, Chita PJ. Fonoaudiologia no método mãe-canguru. In: Hitos SF, Periotto MC. Amamentação: atuação fonoaudiológica; uma abordagem prática e atual. Rio de Janeiro: Revinter; 2009. p.163-71.

5. Ideriha PN, Limongi SCO. Avaliação eletromiográfica da sucção em bebês com síndrome de Down. Rev Soc Bras Fonoaudiol. 2007;12(3):174-83.

6. Xavier C. Avaliação da alimentação de recém-nascidos em fase de hospitalização (escala de avaliação motora oral da Irmandade Santa Casa de Misericórdia de São Paulo). Pró-Fono. 1995;7(2):69-74.

7. Alves AML, Silva EHAA, Oliveira AC. Desmame precoce em prematuros participantes do Método Mãe Canguru. Rev Soc Bras Fonoaudiol. 2007;12(1):23-8.

8. Bühler KEB, Marchi SON. Iniciativa Hospital Amigo da Criança. In: Hitos SF, Periotto MC. Amamentação: atuação fonoaudiológica; uma abordagem prática e atual. Rio de Janeiro: Revinter; 2009. p.173-82.
9. Organização Mundial da Saúde. Organização Pan-Americana da Saúde. Ministério da Saúde (Brasil). Evidências científicas dos dez passos para o sucesso no aleitamento materno. Brasília; OPAS; 2001.

10. Kumar H, Singhal PK, Singh S, Dutta AK, Jain BK, Narayanan I. Spoon vs bottle: a controlled evaluation of milk feeding in young infants. Indian Pediatr. 1989;26(1):11-7.

11. Kuehl J. Cup feeding the newborn: what you should know. J Perinat Neonatal Nurs. 1997;11(2):56-60.

12. Silva SR. Contando com a fonoaudiologia. Rev Assoc Paul Cir Dent. 2004;58(3):167-75.

13. Nagem TM. Chupeta e mamadeira: quem quer, a criança ou seus pais? Rev CEFAC. 1999;1(2):48-55.

14. Affonso DD, Wahlberg V, Persson B. Exploration of mothers' reactions to the kangaroo method of prematurity care. Neonatal Netw. 1989;7(6):43-51.

15. Hurst NM, Valentine CJ, Renfro L, Burns P, Ferlic L. Skin-to-skin holding in the neonatal intensive care unit influences maternal milk volume. J Perinatol. 1997;17(3):213-7.

16. Venâncio SI, Almeida H. Método Mãe Canguru: aplicação no Brasil, evidências científicas e impacto sobre o aleitamento materno. J Pediatr (Rio J). 2004;80(5 Supl):S173-80.

17. Siegel S. Estatística não-paramétrica: para as ciências do comportamento. São Paulo: McGraw-Hill do Brasil; 1975.

18. Gamburgo LJL, Munhoz SRM, Amstalden LG. Alimentação do recém-nascido: aleitamento natural, mamadeira e copinho. Fono Atual. 2002;5(20):39-47. 
Anexo 1. Protocolo de Avaliação e Acompanhamento Fonoaudiológico

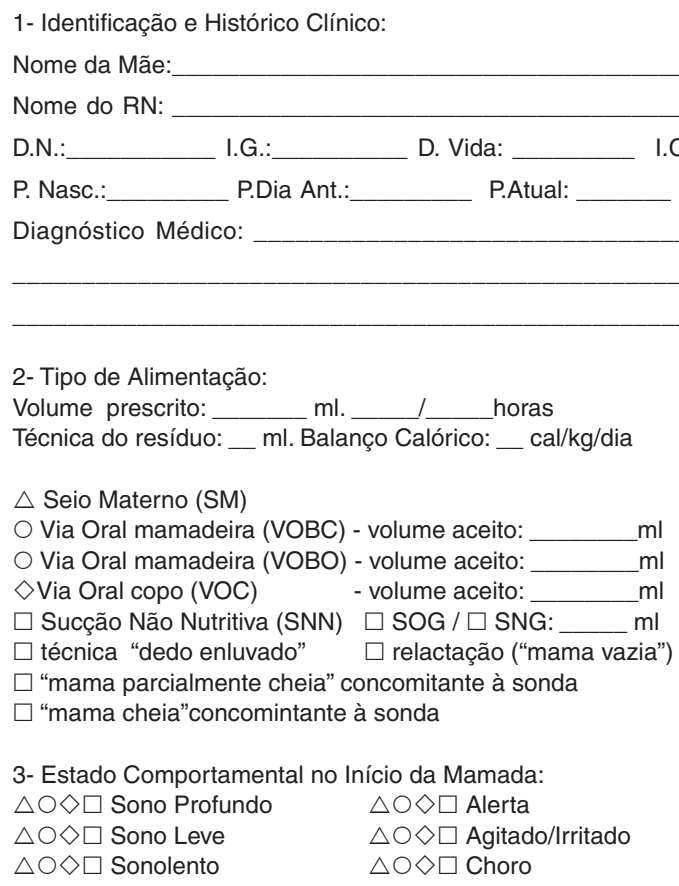

Diagnóstico Médico:

2- Tipo de Alimentação:

Volume prescrito: $\mathrm{ml}$ _ $/$ horas

Técnica do resíduo: _ ml. Balanço Calórico: _ cal $/ \mathrm{kg} / \mathrm{dia}$

$\triangle$ Seio Materno (SM)

Via Oral mamadeira (VOBC) - volume aceito:

$\diamond$ Via Oral copo (VOC) - volume aceito:_ ml

$\square$ Sucção Não Nutritiva (SNN) $\square$ SOG / $\square$ SNG: __ ml

$\square$ técnica "dedo enluvado" $\square$ relactação ("mama vazia")

$\square$ "mama parcialmente cheia" concomitante à sonda

$\square$ "mama cheia"concomintante à sonda

3- Estado Comportamental no Início da Mamada:

$\triangle \bigcirc \diamond \square$ Sono Profundo $\quad \triangle \bigcirc \diamond \square$ Alerta

$\triangle \bigcirc \diamond \square$ Sono Leve $\quad \triangle \bigcirc \diamond \square$ Agitado/Irritado

$\triangle \bigcirc \diamond \square$ Sonolento $\quad \triangle \bigcirc \diamond \square$ Choro

Data:

Horário:
4- Prontidão para a Mamada:

$\triangle \bigcirc \diamond \square$ Movimentos de procura

$\triangle \bigcirc \diamond \square$ Movimentos de sucção

$\triangle \bigcirc \diamond \square$ Leva mãos à face

$\triangle \bigcirc \diamond \square$ Preensão palmar

$\triangle \bigcirc \diamond \square$ Leva mãos à linha média $\triangle \bigcirc \diamond \square$ Protrusão língua

5- Postura Corporal / Padrão Motor

$\triangle \bigcirc \diamond \square$ Estável com apoio $\quad \triangle \bigcirc \diamond \square$ Estável sem apoio

$\triangle \bigcirc \diamond \square$ Flexão $\quad \triangle \bigcirc \diamond \square$ Hipotonia fisiológica

$\triangle \bigcirc \diamond \square$ Instável / Tremores / Desorganização

6- Sucção:

$\triangle \bigcirc \diamond \square$ Não apresenta sucção

$\triangle \bigcirc \diamond \square$ Apresenta grupos de sucções:

$\triangle \bigcirc \diamond \square$ Sucção esporádica

$\triangle \bigcirc \diamond \square$ Regulares

$\triangle \bigcirc \diamond \square$ Irregulares

$\triangle \bigcirc \diamond \square$ Pausas muito longas

$\triangle \bigcirc \diamond \square$ É preciso ajudar a dar pausas $\mathrm{p} / \mathrm{RN}$ se organizar

$\triangle \bigcirc \diamond \square$ Coordenação grupos sucção/deglutição/respiração

7- Grau de Força de Sucção: $\begin{array}{ll}\triangle \bigcirc \diamond \square \text { Fraca } & \triangle \bigcirc \diamond \square \text { Média/Fraca } \\ \triangle \bigcirc \diamond \square \text { Média } & \triangle \bigcirc \diamond \square \text { Forte/Média }\end{array}$

$\triangle \bigcirc \diamond \square$ Forte

8- Variação na Força de Sucção:

$\triangle \bigcirc \diamond \square \operatorname{Sim} \quad \triangle \bigcirc \diamond \square$ Não

9- Variação no Ritmo de Sucção:

$\triangle \bigcirc \diamond \square$ Sim, após $\min$.

$\triangle \bigcirc \diamond \square$ Não $\mathrm{ml}$.

10- Movimentação de Mandíbula:

$\triangle \bigcirc \diamond \square$ Excursão exagerada

$\triangle \bigcirc \diamond \square$ Movimento organizado

$\triangle \bigcirc \diamond \square$ Tremores

$\triangle \bigcirc \diamond \square$ Travamentos

11- Movimentação de língua:

$\triangle \bigcirc \diamond \square$ Retração

$\triangle \bigcirc \diamond \square$ Canolamento

$\triangle \bigcirc \diamond \square$ "Suckling" organizado

$\triangle \bigcirc \diamond \square$ Protrusão exagerada

$\triangle \bigcirc \diamond \square$ Língua alargada

$\triangle \bigcirc \diamond \square$ Incoord. Movimento

12- Deglutição (COPO)

$\diamond$ Lambe a borda do copo

$\diamond$ Introduz a língua no copo

$\diamond$ Engasga ao sorver o leite

$\diamond$ Lambe os lábios

$\diamond$ Sorve o leite c/ coordenação

$\diamond$ Escape de leite ao sorver
13 - Sinais de Estresse:

$\triangle \bigcirc \diamond \square$ Mudança de coloração: $\quad \triangle \bigcirc \diamond \square$ Cianótico $\triangle \bigcirc \diamond \square$ Avermelhado $\triangle \bigcirc \diamond \square$ Taquicardia

$\triangle \bigcirc \diamond \square$ Oscilação da Freq. Cardíaca: $\triangle \bigcirc \diamond \square$ Bradicardia

$\triangle O \diamond \square$ Oscilação / Queda da SAO2 Valor:

$\triangle \bigcirc \diamond \square$ Desconforto Respiratório

$\triangle \bigcirc \diamond \square$ Cansaço. Após__ min. Após

$\triangle \bigcirc \diamond \square$ Escape de leite. Após Após ml.

$\triangle \bigcirc \diamond \square$ Náusea

$\triangle \bigcirc \diamond \square$ Refluxo/ Vômito

$\triangle \bigcirc \diamond \square$ Tosse / Engasgo

14- Outros Sinais de Desconforto:

$\triangle \bigcirc \diamond \square$ Soluços

$\triangle \bigcirc \diamond \square$ Caretas

$\triangle \bigcirc \diamond \square$ Espirros

$\triangle \bigcirc \diamond \square$ Sobressaltos

$\triangle \bigcirc \diamond \square$ Movimentos Corporais de aversão/ recusa

15- Estado Comportamental Após a Mamada:

$\triangle \bigcirc \diamond \square$ Sono Profundo

$\triangle \bigcirc \diamond \square$ Alerta

$\triangle \bigcirc \diamond \square$ Sono Leve

$\triangle \bigcirc \diamond \square$ Sonolento $\triangle \bigcirc \diamond \square$ Agitado/Irritado

Após $\min$ $\mathrm{ml}$. $\triangle \bigcirc \diamond \square$ Choro

16- Horários da Mamada:

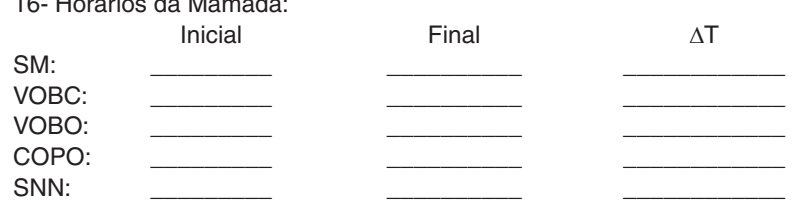

17- Eficácia (VO): $>3 \mathrm{~mL} / \mathrm{min}$, sem sinal de estresse.

$\bigcirc$ Sim $\mathrm{mL} / \mathrm{min}$.

$\bigcirc$ Não $\mathrm{mL} / \mathrm{min}$. $\bigcirc$ Sinal de estresse.

Conduta:

( ) Incentivo à mãe canguru (fase: __ )

( ) Incentivo ao aleitamento materno (SMLD)

( ) Aleitamento materno mais VO, se necessário

( ) VOBC (mamadeira com bico comum)

( ) VOBO (mamadeira com bico ortodôntico)

( ) VOC (copo)

( ) Oferecer dieta exclusivamente por Sonda

( ) Oferecer tudo VO; Sonda, se necessário

( ) Suspender VO

( ) SNN (com "dedo enluvado")

( ) SNN em "mama vazia" (técnica de relactação)

( ) Sucção "mama parcialm/e cheia" concomitante SNG

( ) Seio Materno + _ $\mathrm{ml}$ por sonda

( ) Ausência da mãe: dieta por sonda

( ) Realizar técnica de translactação

( ) Acompanhamento fonoaudiológico

( ) Alta fonoaudiológica

Outros:

Legenda: $\triangle(\mathrm{SM})=$ Seio materno; $\bigcirc(\mathrm{VOBC})$ = Via oral em mamadeira com bico comum; $\bigcirc(\mathrm{VOBO})=$ Via Oral em mamadeira com bico ortodôntico; $\diamond(\mathrm{VOC})=$

Via oral em copo; $\square$ (SNN) = Sucção não Nutritiva; $\square$ SOG = Sonda orogástrica; $\square$ SNG = Sonda nasogástrica; SMLD = seio materno livre demanda

Fonoaudiólogo Responsável:

Auxiliar de enfermagem: 